\title{
T-duality of non-relativistic string in torsional Newton-Cartan background
}

\section{J. Klusoň}

Department of Theoretical Physics and Astrophysics, Faculty of Science, Masaryk University, Kotlářská 2, 611 37, Brno, Czech Republic

E-mail: klu@physics.muni.cz

ABSTRACT: In this short note we analyse T-duality properties of non-relativistic string in torsional Newton-Cartan background. We also determine condition that ensures that non-relativistic string maps to non-relativistic string under T-duality.

KeYwords: Bosonic Strings, String Duality

ARXIV EPRINT: 1909.13508 


\section{Contents}

1 Introduction and summary 1

2 String with light-like isometry and non-relativistic string 2

3 T-duality of non-relativistic string in torsional NC geometry $\quad 6$

\section{Introduction and summary}

Recently there was renewed interest in the Newton-Cartan geometry (NC) and its torsional generalization for the study of non-relativistic aspects of string theory and gravity, see for example [1]. It is interesting that there are currently two versions of non-relativistic string theories. First one was proposed in $[2,3]$ and corresponds to string on torsional NC geometry while the second one was firstly introduced in $[4]^{1}$ and corresponds to string in stringy NC geometry. Stringy NC geometry is characterized by foliation of space-time into two longitudinal directions that, roughly speaking, correspond to world-sheet of fundamental string. In [14] beta function for string in stringy NC gravity was proposed which leads to the dynamical equations of non-relativistic stringy $\mathrm{NC}$ gravity. In case of string on torsional NC geometry beta function was calculated in [15] where again this beta function leads to dynamical equations of motion of torsional NC gravity. This is very interesting consistency check which demonstrates that both non-relativistic string theories could be considered as UV completion of corresponding non-relativistic theories of gravity. Finally it was shown in remarkable paper [16] that these seemingly different string theories can be mapped into each other.

In this work we will be interested in the first version of non-relativistic string theory that arises by null reduction of relativistic string. As was argued in our previous paper [17], following [9] T-duality along null dimension is rather subtle and needs careful treatment. In fact, it is convenient to consider extended string with two auxiliary fields and additional terms on its world-sheet so that the string now propagates in the background with no null isometry. The meaning of two auxiliary fields is that solving their equations of motion and plugging back to the action we get the original one. However since extended action has well defined kinetic term it is much more convenient for performing T-duality as was shown in [17]. We performed it for general null metric and we found that the string in the background with null isometry is T-dual to non-relativistic string.

In this work we apply this procedure to the concrete background [16]. We explicitly derive corresponding Lagrangian density and we found that it has the same form as in [2]. Then we address the main problem which is T-duality of non-relativistic string in torsional

\footnotetext{
${ }^{1}$ For related works, see $[5-8,8-13]$.
} 
$\mathrm{NC}$ background. Since an action for non-relativistic string is non-linear in this background it is not clear whether it is possible to follow standard procedure that is based on the gauging isometry direction on the world-sheet of the string [18, 19]. For that reason we mean that it is natural to start with extended relativistic background and perform T-duality along null direction together with T-duality along one spatial direction. This problem is more complex and we were not able to solve it in the full generality when we have nonzero values of NSNS two form with components along null direction. For that reason we restrict ourselves to the case of zero NSNS two form along these directions leaving the analysis of the most general case to the future. However even in the case of zero NSNS two form we derive interesting results. Explicitly, we show that T-dual string is either relativistic or non-relativistic in accord with the form of the background fields. In more details, in case of the background [16] we find that T-dual string has the same form as the original one when component of the clock form $\tau_{\mu} d x^{\mu}$ along spatial direction $y$, where we perform T-duality, is zero. This result is in agreement with the condition that was derived independently in [23] when T-duality of effective action for non-relativistic D-branes was studied. In the opposite case we obtain ordinary relativistic string in the modified background whose explicit transformation rules are determined by solving equations of motion for two auxiliary fields.

Let us outline our results and suggest possible extension of this work. First of all we applied general analysis that had been studied in [17] to the case of the null background [16] and we derived Lagrangian for non-relativistic string in torsional NC geometry. Then we studied properties of this string under T-duality along spatial direction with isometry and we argued that T-dual string is either relativistic or non-relativistic with dependence on the value of the background clock form $\tau_{\mu} d x^{\mu}$. In case when the T-dual string is again nonrelativistic string in torsional $\mathrm{NC}$ background we found T-dual background fields whose transformation rules are in agreement with Buscher's rules $[18,19]$ and also with the analysis performed in [17] which is nice consistency check of both approaches. On the other hand we should stress that these results were derived on condition when the NSNS two form with components along null and spatial directions are zero. We leave an analysis of the most general case to the future work.

This paper is organized as follows. In the next section 2 we show how Lagrangian for non-relativistic string in torsional NC background can be derived using T-duality transformations along null directions. Then in section 3 we study T-duality of this string along spatial direction and we determine T-dual components of the background fields.

\section{String with light-like isometry and non-relativistic string}

We begin with the bosonic string in the background with null isometry whose dynamics is governed by the Lagrangian density

$$
\begin{aligned}
\mathcal{L}= & -\frac{T}{2} N \sqrt{\omega}\left[-\nabla_{n} x^{\mu} G_{\mu \nu} \nabla_{n} x^{\nu}+\frac{1}{\omega} \partial_{\sigma} x^{\mu} \partial_{\sigma} x^{\nu} G_{\mu \nu}-2 \nabla_{n} x^{\mu} G_{\mu u} \nabla_{n} u+\frac{2}{\omega} \partial_{\sigma} x^{\mu} G_{\mu u} \partial_{\sigma} u\right]- \\
& -T B_{\mu \nu} \partial_{\tau} x^{\mu} \partial_{\sigma} x^{\nu}-T B_{\mu u} \partial_{\tau} x^{\mu} \partial_{\sigma} u-T B_{u \mu} \partial_{\tau} u \partial_{\sigma} x^{\mu}
\end{aligned}
$$


where $T$ is string tension, $G_{\mu \nu}, B_{\mu \nu}, \mu, \nu=0, \ldots, d-1$ are background metric and NSNS two form. Further, $G_{\mu u}$ and $B_{\mu u}$ are background fields along null direction labelled with $u$. Finally we used $1+1$ decomposition of the world-sheet metric where $N$ is two dimensional lapse, $N^{\sigma}$ is two dimensional shift and $\omega$ is spatial component of the metric, and $\nabla_{n}=\frac{1}{N}\left(\partial_{\tau}-N^{\sigma} \partial_{\sigma}\right)$, where $\tau$ and $\sigma$ label time and space dimensions on the string world-sheet, see [17] for more details.

The crucial point which is related to this Lagrangian density is an absence of the metric component $G_{u u}$. This fact makes the Hamiltonian analysis of this theory rather problematic. Then it was shown in [17] that it is convenient to rewrite (2.1) into equivalent form

$$
\begin{aligned}
\mathcal{L}= & -\frac{T}{2} N \sqrt{\omega}\left[-\nabla_{n} x^{\mu} \hat{G}_{\mu \nu} \nabla_{n} x^{\nu}+\frac{1}{\omega} \partial_{\sigma} x^{\mu} \partial_{\sigma} x^{\nu} \hat{G}_{\mu \nu}-2 \nabla_{n} x^{\mu} \hat{G}_{\mu u} \nabla_{n} u+\frac{2}{\omega} \partial_{\sigma} x^{\mu} \hat{G}_{\mu u} \partial_{\sigma} u-\right. \\
& \left.-\nabla_{n} u \hat{G}_{u u} \nabla_{n} u+\frac{1}{\omega} \partial_{\sigma} u \hat{G}_{u u} \partial_{\sigma} u+\lambda^{+} \mathbf{A}+\lambda^{-} \mathbf{B}+\lambda^{+} \lambda^{-}\right]- \\
& -T \hat{B}_{\mu \nu} \nabla_{n} x^{\mu} \partial_{\sigma} x^{\nu}-T \hat{B}_{\mu u} \nabla_{n} x^{\mu} \partial_{\sigma} u-T \hat{B}_{u \nu} \nabla_{n} u \partial_{\sigma} x^{\nu}
\end{aligned}
$$

where now we have to choose $\mathbf{A}$ and $\mathbf{B}$ in such a way to ensure that $\hat{G}_{u u}=0$ after solving equations of motion for $\lambda^{+}$and $\lambda^{-}$. Further, we also demand that $\hat{G}_{\mu \nu}, \hat{B}_{\mu \nu}, \hat{G}_{\mu u}, \hat{B}_{\mu u}$ reduce to $G_{\mu \nu}, B_{\mu \nu}, G_{\mu u}, B_{\mu u}$ when the equations of motion for $\lambda^{+}$and $\lambda^{-}$are solved. More explicitly, the equations of motion for $\lambda^{+}, \lambda^{-}$have the form

$$
\lambda^{-}=-\mathbf{A}, \quad \lambda^{+}=-\mathbf{B}
$$

and hence they give following contribution to the Lagrangian density

$$
\lambda^{+} \mathbf{A}+\lambda^{-} \mathbf{B}+\lambda^{+} \lambda^{-}=-\mathbf{A B}
$$

Generally $\mathbf{A}$ and $\mathbf{B}$ could have the form

$$
\begin{aligned}
& \mathbf{A}=\nabla_{n} x^{\mu} \mathbf{A}_{\mu}+\nabla_{n} u \mathbf{Y}^{+}-\frac{1}{\sqrt{\omega}}\left[\partial_{\sigma} x^{\mu} \mathbf{A}_{\mu}+\partial_{\sigma} y \mathbf{Y}^{+}\right] \\
& \mathbf{B}=\nabla_{n} x^{\mu} \mathbf{B}_{\mu}+\nabla_{n} u \mathbf{Y}^{-}+\frac{1}{\sqrt{\omega}}\left[\partial_{\sigma} x^{\mu} \mathbf{B}_{\mu}+\partial_{\sigma} u \mathbf{Y}^{-}\right] .
\end{aligned}
$$

However as was shown in [17] it is sufficient to consider the case when $\mathbf{A}_{\mu}=\mathbf{B}_{\mu}=0$ since their non-zero values modify the background metric $G_{\mu \nu}$ only. In this case we obtain following contribution to the Lagrangian after solving equations of motion for $\lambda^{+}, \lambda^{-}$in the form

$$
-\mathbf{A B}=-\left[\nabla_{n} u \nabla_{n} u-\frac{1}{\omega} \partial_{\sigma} u \partial_{\sigma} u\right] \mathbf{Y}^{+} \mathbf{Y}^{-}
$$

which implies that the Lagrangian density (2.2) reduces to the original one when

$$
\mathbf{Y}^{+}=\sqrt{\hat{G}_{u u}}, \quad \mathbf{Y}^{-}=-\sqrt{\hat{G}_{u u}}
$$


Note also that in this case all hatted and unhatted components of the background fields coincide. In other words, an extended action has the form

$$
\begin{aligned}
\mathcal{L}= & -\frac{T}{2} N \sqrt{\omega}\left[-\nabla_{n} x^{\mu} \hat{G}_{\mu \nu} \nabla_{n} x^{\nu}+\frac{1}{\omega} \partial_{\sigma} x^{\mu} \partial_{\sigma} x^{\nu} \hat{G}_{\mu \nu}-2 \nabla_{n} x^{\mu} \hat{G}_{\mu u} \nabla_{n} u+\frac{2}{\omega} \partial_{\sigma} x^{\mu} \hat{G}_{\mu u} \partial_{\sigma} u-\right. \\
& -\nabla_{n} u \hat{G}_{u u} \nabla_{n} u+\frac{1}{\omega} \partial_{\sigma} u \hat{G}_{u u} \partial_{\sigma} u+ \\
& \left.+\lambda^{+}\left(\nabla_{n} u-\frac{1}{\sqrt{\omega}} \partial_{\sigma} u\right) \mathbf{Y}^{+}+\lambda^{-}\left(\nabla_{n} u+\frac{1}{\sqrt{\omega}} \partial_{\sigma} u\right) \mathbf{Y}^{-}+\lambda^{+} \lambda^{-}\right]- \\
& -T \hat{B}_{\mu \nu} \nabla_{n} x^{\mu} \partial_{\sigma} x^{\nu}-T \nabla_{n} x^{\mu} \hat{B}_{\mu u} \partial_{\sigma} u-\nabla_{n} u \hat{B}_{u \nu} \partial_{\sigma} x^{\nu} .
\end{aligned}
$$

This action is the starting point of our analysis which is based on the canonical description of T-duality. The Hamiltonian corresponding to the action (2.8) was found in [17] and has the form

$$
\begin{aligned}
H= & \int d \sigma\left(N^{\tau} \mathcal{H}_{\tau}+N^{\sigma} \mathcal{H}_{\sigma}\right) \\
\mathcal{H}_{\tau}= & \pi_{\mu} \hat{G}^{\mu \nu} \pi_{\nu}+2 \pi_{\mu} \hat{G}^{\mu u}\left(\pi_{u}+\frac{T}{2} \tilde{\lambda}^{+} \mathbf{Y}^{+}+\frac{T}{2} \tilde{\lambda}^{-} \mathbf{Y}^{-}\right)+ \\
& +\left(\pi_{u}+\frac{T}{2} \tilde{\lambda}^{+} \mathbf{Y}^{+}+\frac{T}{2} \tilde{\lambda}^{-} \mathbf{Y}^{-}\right) \hat{G}^{u u}\left(\pi_{u}+\frac{T}{2} \tilde{\lambda}^{+} \mathbf{Y}^{+}+\frac{T}{2} \tilde{\lambda}^{-} \mathbf{Y}^{-}\right)+ \\
& +T^{2} \partial_{\sigma} x^{\mu} \hat{G}_{\mu \nu} \partial_{\sigma} x^{\nu}+2 T^{2} \partial_{\sigma} x^{\mu} \hat{G}_{\mu u} \partial_{\sigma} u+T^{2} \partial_{\sigma} u \hat{G}_{u u} \partial_{\sigma} u- \\
& -T^{2} \tilde{\lambda}^{+} \partial_{\sigma} u \mathbf{Y}^{+}+T^{2} \tilde{\lambda}^{-} \partial_{\sigma} u \mathbf{Y}^{-}+T^{2} \tilde{\lambda}^{+} \tilde{\lambda}^{-}, \quad \mathcal{H}_{\sigma}=p_{\mu} \partial_{\sigma} x^{\mu}+p_{u} \partial_{\sigma} u
\end{aligned}
$$

where we performed rescaling

$$
\sqrt{\omega} \lambda^{+}=\tilde{\lambda}^{+}, \quad \sqrt{\omega} \lambda^{-}=\tilde{\lambda}^{-}
$$

and where $p_{\mu}$ and $p_{u}$ are momenta conjugate to $x^{\mu}, u$ respectively and where

$$
\pi_{\mu}=p_{\mu}+T \hat{B}_{\mu \nu} \partial_{\sigma} x^{\nu}+T \hat{B}_{\mu u} \partial_{\sigma} u, \quad \pi_{u}=p_{u}+T \hat{B}_{u \mu} \partial_{\sigma} x^{\mu} .
$$

Having identified canonical Hamiltonian we can proceed to the definition of nonrelativistic string when we perform T-duality along $u$ direction. This is done when we introduce dual coordinate $\eta$ and $p_{\eta}$ that are related to $p_{u}$ and $u$ by canonical transformations $[20,21]$

$$
p_{u}=-T \partial_{\sigma} \eta, \quad p_{\eta}=-T \partial_{\sigma} u .
$$

Then performing again inverse Legendre transformation to Lagrangian description we obtain T-dual Lagrangian in the form

$$
\begin{aligned}
\mathcal{L}^{\prime}= & \frac{1}{4 N^{\tau}}\left(g_{\tau \tau}^{\prime}-2 N^{\sigma} g_{\tau \sigma}^{\prime}+\left(N^{\sigma}\right)^{2} g_{\sigma \sigma}^{\prime}\right)-N^{\tau} T^{2} g_{\sigma \sigma}^{\prime}-T \hat{B}_{M N}^{\prime} \partial_{\tau} \tilde{x}^{M} \partial_{\sigma} \tilde{x}^{N}+ \\
& +\frac{T}{2} N \tilde{\lambda}^{+}\left(\nabla_{n} \tilde{x}^{M} \mathbf{A}_{M}-2 T \partial_{\sigma} \tilde{x}^{M} \mathbf{A}_{M}\right)+\frac{T}{2} N \tilde{\lambda}^{-}\left(\nabla_{n} \tilde{x}^{M} \mathbf{B}_{M}+2 T \partial_{\sigma} \tilde{x}^{M} \mathbf{B}_{M}\right),
\end{aligned}
$$

where

$$
g_{\alpha \beta}^{\prime}=\hat{G}_{M N}^{\prime} \partial_{\alpha} \tilde{x}^{M} \partial_{\beta} \tilde{x}^{N}, \quad \tilde{x}^{M} \equiv\left(x^{\mu}, \eta\right)
$$


and where the background fields are given by standard Buscher's rules [18, 19]

$$
\begin{aligned}
& \hat{G}_{\mu \nu}^{\prime}=\hat{G}_{\mu \nu}-\frac{1}{\hat{G}_{u u}} \hat{G}_{\mu u} \hat{G}_{u \nu}+\frac{1}{\hat{G}_{u u}} \hat{B}_{\mu u} \hat{B}_{\nu u}, \\
& \hat{G}_{\mu \eta}^{\prime}=\frac{\hat{B}_{\mu u}}{\hat{G}_{u u}}, \quad \hat{G}_{\eta \mu}^{\prime}=-\frac{\hat{B}_{u \nu}}{\hat{G}_{u u}}, \quad \hat{G}_{\eta \eta}^{\prime}=\frac{1}{\hat{G}_{u u}} \\
& \hat{B}_{\mu \nu}^{\prime}=\hat{B}_{\mu \nu}-\frac{\hat{G}_{\mu u}}{\hat{G}_{u u}} \hat{B}_{u \nu}-\frac{\hat{B}_{\mu u}}{\hat{G}_{u u}} \hat{G}_{u \nu}, \\
& \hat{B}_{\mu \eta}^{\prime}=\frac{\hat{G}_{\mu \eta}}{\hat{G}_{u u}}, \quad \hat{B}_{\eta \nu}^{\prime}=-\frac{\hat{G}_{u \nu}}{\hat{G}_{u u}} .
\end{aligned}
$$

Finally, $\mathbf{A}_{M}$ and $\mathbf{B}_{M}$ are defined as

$$
\mathbf{A}_{M}=\left(\frac{1}{\sqrt{\hat{G}_{u u}}}\left(\hat{G}_{\mu u}-\hat{B}_{\mu u}\right),-\frac{1}{\sqrt{\hat{G}_{u u}}}\right), \quad \mathbf{B}_{M}=-\left(\frac{1}{\sqrt{\hat{G}_{u u}}}\left(\hat{G}_{\mu u}+\hat{B}_{\mu u}\right), \frac{1}{\sqrt{\hat{G}_{u u}}}\right) .
$$

Let us now solve the equations of motion for $\tilde{\lambda}^{+}, \tilde{\lambda}^{-}$that have the form

$$
\nabla_{n} \tilde{x}^{M} \mathbf{A}_{M}=2 T \partial_{\sigma} \tilde{x}^{M} \mathbf{A}_{M}, \quad \nabla_{n} \tilde{x}^{M} \mathbf{B}_{M}=-2 T \partial_{\sigma} \tilde{x}^{M} \mathbf{B}_{M} .
$$

If we multiply the first equation with $\partial_{\sigma} \tilde{x}^{M} \mathbf{B}_{M}$ and the second one by $\partial_{\sigma} \tilde{x}^{N} \mathbf{A}_{N}$ and sum them we obtain

$$
N^{\sigma}=\frac{\mathbf{M}_{\tau \sigma}}{\mathbf{M}_{\sigma \sigma}}, \quad \mathbf{M}_{\alpha \beta}=\partial_{\alpha} \tilde{x}^{M} \mathbf{M}_{M N} \partial_{\beta} \tilde{x}^{N},
$$

where the matrix $\mathbf{M}_{M N}$ is defined as

$$
\mathbf{M}_{M N}=\frac{1}{2}\left(\mathbf{A}_{M} \mathbf{B}_{N}+\mathbf{B}_{M} \mathbf{A}_{N}\right)
$$

Further, if we multiply two equations in (2.17) we obtain

$$
N^{\tau}=\frac{\sqrt{-\operatorname{det} \mathbf{M}_{\alpha \beta}}}{2 \mathbf{M}_{\sigma \sigma}} .
$$

Then the final Lagrangian density has the form of non-relativistic string action

$$
\mathcal{L}^{T}=-\frac{T}{2} \sqrt{-\operatorname{det} \mathbf{M}} \mathbf{M}^{\alpha \beta} g_{\alpha \beta}^{\prime}-T \hat{B}_{\mu \nu}^{\prime} \partial_{\tau} \tilde{x}^{\mu} \partial_{\sigma} \tilde{x}^{\nu}-T \hat{B}_{\mu \eta}^{\prime} \partial_{\tau} x^{\mu} \partial_{\sigma} \eta-T \hat{B}_{\eta \mu}^{\prime} \partial_{\tau} \eta \partial_{\sigma} x^{\mu},
$$

where $\mathbf{M}^{\alpha \beta}$ is matrix inverse to $\mathbf{M}_{\alpha \beta}$ so that $\mathbf{M}^{\alpha \beta} \mathbf{M}_{\beta \gamma}=\delta_{\beta}^{\alpha}$.

We use this general procedure for the case of the background with null isometry which defines $\mathrm{NC}$ with torsion $[2,16]$

$$
d s^{2}=g_{M N} d x^{M} d x^{N}=2 \tau(d u-m)+h_{\mu \nu} d x^{\mu} d x^{\nu}, \quad \tau=\tau_{\mu} d x^{\mu}, \quad m=m_{\mu} d x^{\mu},
$$

where $\operatorname{det} h_{\mu \nu}=0$. We also have non-zero NSNS two form with following components

$$
\hat{B}_{\mu \nu}, \quad \hat{B}_{u \mu}=b_{\mu} .
$$


For this background the components of the matrix $\mathbf{M}_{M N}$ have the form

$$
\mathbf{M}_{\mu \nu}=-\frac{1}{\hat{G}_{u u}}\left(\tau_{\mu} \tau_{\nu}-b_{\mu} b_{\nu}\right), \quad \mathbf{M}_{\mu \eta}=-\frac{b_{\mu}}{\hat{G}_{u u}}, \quad \mathbf{M}_{\eta \eta}=\frac{1}{\hat{G}_{u u}} .
$$

Without loss of generality we can take $\hat{G}_{u u}=1$ and hence we obtain

$$
\mathbf{M}_{\alpha \beta}=\left(b_{\alpha}-\partial_{\alpha} \eta\right)\left(b_{\beta}-\partial_{\beta} \eta\right)-\tau_{\alpha} \tau_{\beta}
$$

so that

$$
\operatorname{det} \mathbf{M}=-\left(\left(\partial_{\tau} \eta-b_{\tau}\right) \tau_{\sigma}-\tau_{\tau}\left(\partial_{\sigma} \eta-b_{\sigma}\right)\right)^{2} .
$$

Further, with the help of the background fields (2.21) we obtain

$$
\begin{aligned}
& \hat{g}_{\alpha \beta}^{\prime}=\hat{h}_{\alpha \beta}-\tau_{\alpha} \tau_{\beta}+\left(\partial_{\alpha} \eta-b_{\alpha}\right)\left(\partial_{\beta} \eta-b_{\beta}\right), \\
& \hat{B}_{\mu \nu}^{\prime}=\hat{B}_{\mu \nu}-\tau_{\mu} b_{\nu}+\tau_{\nu} b_{\mu}
\end{aligned}
$$

so that we obtain

$$
\hat{B}_{\mu \nu}^{\prime} \partial_{\tau} x^{\mu} \partial_{\sigma} x^{\nu}+\hat{B}_{\mu \eta}^{\prime} \partial_{\tau} x^{\mu} \partial_{\sigma} \eta+\hat{B}_{\eta \mu}^{\prime} \partial_{\tau} \eta \partial_{\sigma} x^{\mu}=\hat{B}_{\tau \sigma}+\tau_{\sigma}\left(b_{\tau}-\partial_{\tau} \eta\right)-\tau_{\tau}\left(b_{\sigma}-\partial_{\sigma} \eta\right),
$$

where $\hat{h}_{\alpha \beta}=\hat{h}_{\mu \nu} \partial_{\alpha} x^{\mu} \partial_{\beta} x^{\nu}, \hat{h}_{\mu \nu}=h_{\mu \nu}-\tau_{\mu} m_{\nu}-\tau_{\nu} m_{\mu}$. Then we see that Lagrangian density is equal to

$$
\begin{aligned}
\mathcal{L}= & \frac{T}{2\left(\left(\partial_{\tau} \eta-b_{\tau}\right) \tau_{\sigma}-\tau_{\tau}\left(\partial_{\sigma} \eta-b_{\sigma}\right)\right)} \times \\
& \times\left[\left(\left(\partial_{\sigma} \eta-b_{\sigma}\right)\left(\partial_{\sigma} \eta-b_{\sigma}\right)-\tau_{\sigma} \tau_{\sigma}\right) \hat{h}_{\tau \tau}-2\left(\left(\partial_{\sigma} \eta-b_{\sigma}\right)\left(\partial_{\tau} \eta-b_{\tau}\right)-\tau_{\sigma} \tau_{\tau}\right) \hat{h}_{\tau \sigma}+\right. \\
& \left.+\left(\left(\partial_{\tau} \eta-b_{\tau}\right)\left(\partial_{\tau} \eta-b_{\tau}\right)-\tau_{\tau} \tau_{\tau}\right) \hat{h}_{\sigma \sigma}\right] \\
= & \frac{T}{2\left(\left(\partial_{\tau} \eta-b_{\tau}\right) \tau_{\sigma}-\tau_{\tau}\left(\partial_{\sigma} \eta-b_{\sigma}\right)\right.} \epsilon^{\alpha \alpha^{\prime}} \epsilon^{\beta \beta^{\prime}}\left(\left(\partial_{\alpha} \eta-b_{\alpha}\right)\left(\partial_{\beta} \eta-b_{\beta}\right)-\tau_{\alpha} \tau_{\beta}\right) \hat{h}_{\alpha^{\prime} \beta^{\prime}}
\end{aligned}
$$

were $\epsilon^{\alpha \beta}=-\epsilon^{\beta \alpha}, \epsilon^{01}=1$. We see that the Lagrangian density (2.27) agrees with the Lagrangian density found in [16] and we mean that this is nice consistency check of the general procedure outlined above. In the next section we analyse how this non-relativistic string transforms under T-duality.

\section{T-duality of non-relativistic string in torsional NC geometry}

As was shown in [16] the Lagrangian density (2.27) corresponds to Nambu-Gotto form of the action for non-relativistic string in torsional $\mathrm{NC}$ background. It is well known that such an action, due to its non-linearity is not well suitable for the study of T-duality based on the gauging of the isometry direction. For that reason we mean that it is natural to start with extended relativistic string and perform T-duality along both two directions, one corresponding to the original dimension that defines non-relativistic string and the second one that corresponds to T-duality along spatial dimension. Since we study this problem with the help of the canonical formalism we start with the Hamiltonian for extended string 
and perform T-duality along two directions, one corresponding to $u$ and the second one to spatial direction that we label as $y$. We use common notation where

$$
\tilde{p}^{m}=\left(p_{\tilde{y}}, p_{\eta}\right), \quad \tilde{x}_{m}=(\tilde{y}, \eta), m=\tilde{y}, \eta .
$$

Finally we define $\mathbf{Y}_{m}^{ \pm}$as $\mathbf{Y}_{m}^{ \pm}=\left(0, \mathbf{Y}^{ \pm}\right)$. We also restrict ourselves to the case when $\hat{B}_{\mu u}=0$. Then T-dual Hamiltonian constraint has the form

$$
\begin{aligned}
\mathcal{H}_{\tau}^{T}= & \mathcal{H}_{\tau}\left(p_{y}=-T \partial_{\sigma} \tilde{y}, p_{u}=-T \partial_{\sigma} \eta, \partial_{\sigma} y=-T^{-1} p_{\tilde{y}}, \partial_{\sigma} u=-T^{-1} p_{\eta}\right)= \\
= & \left(k_{i}^{\prime}-\hat{B}_{i m} \tilde{p}^{m}\right) \hat{G}^{i j}\left(k_{j}^{\prime}-\hat{B}_{j n} \tilde{p}^{n}\right)+ \\
& -2 T\left(k_{i}^{\prime}-\hat{B}_{i m} \tilde{p}^{m}\right) \hat{G}^{i n} \mathbf{W}_{n}+T^{2} \mathbf{W}_{m} \hat{G}^{m n} \mathbf{W}_{n}+ \\
& +T\left(k_{i}^{\prime}-\hat{B}_{i n} \tilde{p}^{n}\right) \hat{G}^{i m}\left(\tilde{\lambda}^{+} \mathbf{Y}_{m}^{+}+\tilde{\lambda}^{-} \mathbf{Y}_{m}^{-}\right)-T^{2} \mathbf{W}_{m} \hat{G}^{m n}\left(\tilde{\lambda}^{+} \mathbf{Y}_{n}^{+}+\tilde{\lambda}^{-} \mathbf{Y}_{n}^{-}\right)+ \\
& +\frac{T^{2}}{4}\left(\tilde{\lambda}^{+} \mathbf{Y}_{m}^{+}+\tilde{\lambda}^{-} \mathbf{Y}_{m}^{-}\right) \hat{G}^{m n}\left(\tilde{\lambda}^{+} \mathbf{Y}_{n}^{+}+\tilde{\lambda}^{-} \mathbf{Y}_{n}^{-}\right)+T \tilde{\lambda}^{+} \tilde{p}^{m} \mathbf{Y}_{m}^{+}-T \tilde{\lambda}^{-} \tilde{p}^{m} \mathbf{Y}_{m}^{-}+T^{2} \tilde{\lambda}^{+} \tilde{\lambda}^{-}+ \\
& +T^{2} \partial_{\sigma} x^{i} \hat{G}_{i j} \partial_{\sigma} x^{j}-2 T \partial_{\sigma} x^{i} \hat{G}_{i m} \tilde{p}^{m}+\tilde{p}^{m} \hat{G}_{m n} \tilde{p}^{n}, \\
\mathcal{H}_{\sigma}^{T}= & p_{i} \partial_{\sigma} x^{i}+p_{\tilde{y}} \partial_{\sigma} \tilde{y}+p_{\eta} \partial_{\sigma} \eta,
\end{aligned}
$$

where $k_{i}^{\prime}=p_{i}+T \hat{B}_{i j} \partial_{\sigma} x^{j}, i, j, k=0, \ldots, d-2$ and where

$$
\mathbf{W}_{m}=\partial_{\sigma} \tilde{x}_{m}-\hat{B}_{m i} \partial_{\sigma} x^{i} .
$$

In order to determine form of the background fields it is convenient to derive corresponding Lagrangian from (3.2). We begin with the equations of motion for $\tilde{x}_{m}, x^{i}$

$$
\begin{aligned}
\partial_{\tau} x^{i}= & \left\{x^{i}, H^{T}\right\}=2 N^{\tau} \hat{G}^{i j}\left(k_{j}^{\prime}-\hat{B}_{j m} \tilde{p}^{m}\right)-2 T N^{\tau} \hat{G}^{i m} \mathbf{W}_{m}+ \\
& +T N^{\tau} \hat{G}^{i m}\left(\tilde{\lambda}^{+} \mathbf{Y}_{m}^{+}+\tilde{\lambda}^{-} \mathbf{Y}_{m}^{-}\right)+N^{\sigma} \partial_{\sigma} x^{i} \\
\partial_{\tau} \tilde{x}_{m}= & \left\{\tilde{x}_{m}, H^{T}\right\}=2 N^{\tau} \hat{B}_{m i} \hat{G}^{i j}\left(k_{j}-\hat{B}_{j n} \tilde{p}^{n}\right)-2 T N^{\tau} \hat{B}_{m i} \hat{G}^{i n} \mathbf{W}_{n}+ \\
& +T N^{\tau} \hat{B}_{m i} \hat{G}^{i n}\left(\tilde{\lambda}^{+} \mathbf{Y}_{n}^{+}+\tilde{\lambda}^{-} \mathbf{Y}_{n}^{-}\right)+T N^{\tau}\left(\tilde{\lambda}^{+} \mathbf{Y}_{m}^{+}-\tilde{\lambda}^{-} \mathbf{Y}_{m}^{-}\right) \\
& -2 T N^{\tau} \hat{G}_{m i} \partial_{\sigma} x^{i}+2 N^{\tau} \hat{G}_{m n} \tilde{p}^{n}+N^{\sigma} \partial_{\sigma} \tilde{x}_{m},
\end{aligned}
$$

where $H^{T}=\int d \sigma\left(N^{\tau} \mathcal{H}_{\tau}^{T}+N^{\sigma} \mathcal{H}_{\sigma}^{T}\right)$. If we combine (3.4) together we can express $\tilde{p}^{m}$ as

$$
\tilde{p}^{m}=\frac{1}{2 N^{\tau}} \tilde{G}^{m n}\left(\tilde{X}_{n}-\hat{B}_{n i} X^{i}+2 T N^{\tau} \hat{G}_{n i} \partial_{\sigma} x^{i}-T N^{\tau}\left(\tilde{\lambda}^{+} \mathbf{Y}_{n}^{+}-\tilde{\lambda}^{-} \mathbf{Y}_{n}^{-}\right)\right)
$$

where $\tilde{X}_{n}=\partial_{\tau} \tilde{x}_{n}-N^{\sigma} \partial_{\sigma} \tilde{x}_{n}, X^{i}=\partial_{\tau} x^{i}-N^{\sigma} \partial_{\sigma} x^{i}$ and where we introduced matrix inverse $\tilde{G}^{m n}$ to $\hat{G}_{m n}$

$$
\tilde{G}^{m n} \hat{G}_{n p}=\delta_{p}^{m} .
$$

We further introduce matrix $\tilde{G}_{i j}$ inverse to $\hat{G}^{i j}$

$$
\tilde{G}_{i j}=\hat{G}_{i j}-\hat{G}_{i m} \tilde{G}^{m n} \hat{G}_{m j}, \quad \tilde{G}_{i j} \hat{G}^{j k}=\delta_{i}^{k}, \quad \tilde{G}_{i j} \hat{G}^{j m}=-\hat{G}_{i n} \tilde{G}^{n m}
$$

so that

$$
k_{i}^{\prime}-\hat{B}_{i m} \tilde{p}^{m}=\frac{1}{2 N^{\tau}} \tilde{G}_{i j}\left(X^{j}+2 T N^{\tau} \hat{G}^{j m} \mathbf{W}_{m}-T N^{\tau} \hat{G}^{j m}\left(\tilde{\lambda}^{+} \mathbf{Y}_{m}^{+}+\tilde{\lambda}^{-} \mathbf{Y}_{m}^{-}\right)\right) .
$$


Then, after some algebra, we find Lagrangian density in the form

$$
\begin{aligned}
\mathcal{L}^{T}= & \tilde{p}^{m} \partial_{\tau} \tilde{x}_{m}+p_{i} \partial_{\tau} x^{i}-N^{\tau} \mathcal{H}_{\tau}^{T}-N^{\sigma} \mathcal{H}_{\sigma}^{T}= \\
= & \frac{1}{4 N^{\tau}}\left(X^{i} \hat{G}_{i j}^{\prime} X^{j}+\tilde{X}_{m} \hat{G}^{\prime m n} \tilde{X}_{n}+\tilde{X}_{m} \hat{G}_{i}^{\prime m} X^{i}+X^{i} \hat{G}_{i}^{\prime}{ }^{n} \tilde{X}_{n}\right)- \\
& -N^{\tau} T^{2}\left(\partial_{\sigma} \tilde{x}_{m} \hat{G}^{\prime m n} \partial_{\sigma} \tilde{x}_{n}+\partial_{\sigma} \tilde{x}_{m} \hat{G}_{i}^{\prime m} \partial_{\sigma} x^{i}+\partial_{\sigma} x^{i} \hat{G}_{i}^{\prime}{ }^{m} \partial_{\sigma} \tilde{x}_{m}+\partial_{\sigma} x^{i} \hat{G}_{i j}^{\prime} \partial_{\sigma} x^{j}\right)- \\
& -T \hat{B}_{i j}^{\prime} \partial_{\tau} x^{i} \partial_{\sigma} x^{j}-T \hat{B}_{i}^{\prime} \partial_{\tau} x^{i} \partial_{\sigma} \tilde{x}_{m}-T \hat{B}_{i}^{\prime m} \partial_{\tau} \tilde{x}_{m} \partial_{\sigma} x^{i}+ \\
& -\frac{T}{2} N^{\tau} \tilde{\lambda}^{+}\left(\nabla_{n} x^{i}\left(-\tilde{G}_{i m}+\hat{B}_{i m}\right) \hat{G}^{m n} \mathbf{Y}_{n}^{+}+\nabla_{n} \tilde{x}_{n} \tilde{G}^{n m} \mathbf{Y}_{m}^{+}-\right. \\
& \left.-2 \partial_{\sigma} \tilde{x}_{m} \tilde{G}^{m n} \mathbf{Y}_{n}^{+}+2 \partial_{\sigma} x^{i}\left(\hat{G}_{i n}-\hat{B}_{i n}\right) \tilde{G}^{m n} \mathbf{Y}_{n}^{+}\right) \\
& -\frac{T}{2} N^{\tau} \tilde{\lambda}^{-}\left(\nabla_{n} x^{i}\left(-\tilde{G}_{i m}-\hat{B}_{i m}\right) \tilde{G}^{m n} \mathbf{Y}_{n}^{-}-\nabla_{n} \tilde{x}_{n} \tilde{G}^{n m} \mathbf{Y}_{m}^{-}-\right. \\
& \left.-2 \partial_{\sigma} \tilde{x}_{n} \tilde{G}^{n m} \mathbf{Y}_{m}^{-}-2 \partial_{\sigma} x^{i}\left(\hat{G}_{i m}+\hat{B}_{i m}\right) \tilde{G}^{m n} \mathbf{Y}_{n}^{-}\right)- \\
& -T^{2} N^{\tau} \tilde{\lambda}^{+} \tilde{\lambda}^{-}\left(\mathbf{Y}_{m}^{+} \tilde{G}^{m n} \mathbf{Y}_{n}^{-}+1\right),
\end{aligned}
$$

where we have following components of background metric and NSNS two form

$$
\begin{aligned}
\hat{G}_{i j}^{\prime} & =\tilde{G}_{i j}-\hat{B}_{i n} \tilde{G}^{n m} \hat{B}_{m j}=\hat{G}_{i j}-\hat{G}_{i n} \tilde{G}^{n m} \hat{G}_{n j}-\hat{B}_{i n} \tilde{G}^{n m} \hat{B}_{m j}, \\
\hat{G}^{\prime m n} & =\tilde{G}^{m n}, \quad \hat{G}_{i}^{\prime m}=-\tilde{G}^{m n} \hat{B}_{n i}, \quad \hat{G}_{i}^{\prime n}=\hat{B}_{i m} \tilde{G}^{m n}, \\
\hat{B}_{i}^{\prime m} & =\hat{G}_{i n} \tilde{G}^{n m}, \quad \hat{B}_{i}^{\prime m}=-\hat{G}^{m n} \hat{G}_{m i}, \quad \hat{B}_{i j}^{\prime}=\hat{B}_{i j}-\hat{B}_{i n} \tilde{G}^{n m} \hat{G}_{m j}+\hat{G}_{i m} \tilde{G}^{m n} \hat{B}_{n j} .
\end{aligned}
$$

Now the nature of T-dual string depends on the form of the inverse matrix $\tilde{G}^{m n}$. In case when $\tilde{G}^{\eta \eta}=\frac{1}{\hat{G}_{u u}}$ we obtain that T-dual string is non-relativistic string. To see this we introduce again notation

$$
\begin{aligned}
\tilde{x}^{M} & =\left(x^{i}, \tilde{x}_{m}\right), \quad \mathbf{A}_{M}=\left(\left(-\hat{G}_{i n}+\hat{B}_{i n}\right) \tilde{G}^{n m} \mathbf{Y}_{m}^{+}, \tilde{G}^{n m} \mathbf{Y}_{m}^{+}\right), \\
\mathbf{B}_{M} & =\left(\left(-\hat{G}_{i n}-\hat{B}_{i n}\right) \tilde{G}^{n m} \mathbf{Y}_{m}^{-},-\tilde{G}^{n m} \mathbf{Y}_{m}^{-}\right),
\end{aligned}
$$

where of course we could express $\mathbf{A}_{M}$ and $\mathbf{B}_{M}$ with the help of the transformed fields given in (3.10). Then the expression proportional to $\tilde{\lambda}^{+}, \tilde{\lambda}^{-}$can be written as

$$
\begin{aligned}
& -\frac{T}{2} N^{\tau} \tilde{\lambda}^{+}\left(\nabla_{n} \tilde{x}^{M} \mathbf{A}_{M}-2 T \partial_{\sigma} \tilde{x}^{M} \mathbf{A}_{M}\right)- \\
& -\frac{T}{2} N^{\tau} \tilde{\lambda}^{+}\left(\nabla_{n} \tilde{x}^{M} \mathbf{B}_{M}+2 T \partial_{\sigma} \tilde{x}^{M} \mathbf{B}_{M}\right)-T^{2} N^{\tau} \tilde{\lambda}^{+} \tilde{\lambda}^{-}\left(\mathbf{Y}_{m}^{+} \tilde{G}^{m n} \mathbf{Y}_{n}^{-}+1\right) .
\end{aligned}
$$

Now in the first case when $\tilde{G}^{\eta \eta}=\frac{1}{\hat{G}_{u u}}$ we find that $\mathbf{Y}_{m}^{+} \tilde{G}^{m n} \mathbf{Y}_{n}^{-}+1=0$ and hence we find

$$
N^{\sigma}=\frac{\mathbf{M}_{\tau \sigma}}{\mathbf{M}_{\sigma \sigma}}, \quad N=\frac{\sqrt{-\operatorname{det} \mathbf{M}_{\alpha \beta}}}{2 \mathbf{M}_{\sigma \sigma}},
$$

where

$$
\mathbf{M}_{\alpha \beta}=\partial_{\alpha} \tilde{x}^{M} \mathbf{M}_{M N} \partial_{\beta} \tilde{x}^{N}, \quad \mathbf{M}_{M N}=\frac{1}{2}\left(\mathbf{A}_{M} \mathbf{B}_{N}+\mathbf{B}_{M} \mathbf{A}_{N}\right) .
$$


As a result we obtain Lagrangian density in the form

$$
\mathcal{L}^{T}=-\frac{T}{2} \sqrt{-\operatorname{det} \mathbf{M}} \mathbf{M}^{\alpha \beta} \hat{G}_{M N}^{\prime} \partial_{\alpha} \tilde{x}^{M} \partial_{\beta} \tilde{x}^{N}-T \hat{B}_{M N}^{\prime} \partial_{\tau} \tilde{x}^{M} \partial_{\sigma} \tilde{x}^{N} .
$$

Let us now concentrate on situation when $\mathbf{Y}_{m}^{+} \tilde{G}^{m n} \mathbf{Y}_{n}^{-}+1 \neq 0$ and denote $\mathbf{X}$ as $\mathbf{X}=\left(\mathbf{Y}_{m}^{+} \tilde{G}^{m n} \mathbf{Y}_{n}^{-}+1\right)$. Then the equation of motion for $\tilde{\lambda}^{ \pm}$can be solved as

$$
\tilde{\lambda}^{-}=-\frac{1}{2 T \mathbf{X}}\left(\nabla_{n} \tilde{x}^{M} \mathbf{A}_{M}-2 T \partial_{\sigma} \tilde{x}^{M} \mathbf{A}_{M}\right), \quad \tilde{\lambda}^{+}=-\frac{1}{2 T \mathbf{X}}\left(\nabla_{n} \tilde{x}^{M} \mathbf{B}_{M}+2 T \partial_{\sigma} \tilde{x}^{M} \mathbf{B}_{M}\right) .
$$

Inserting this result into (3.12) we obtain following contribution to the Lagrangian density (3.9)

$$
\frac{1}{2 \mathbf{X}} N^{\tau}\left(\nabla_{n} \tilde{x}^{M} \mathbf{A}_{M}-2 T \partial_{\sigma} \tilde{x}^{M} \mathbf{A}_{M}\right)\left(\nabla_{n} \tilde{x}^{N} \mathbf{B}_{N}+2 T \partial_{\sigma} \tilde{x}^{N} \mathbf{B}_{N}\right)
$$

and hence we obtain relativistic form of the Lagrangian density

$$
\mathcal{L}=\frac{1}{4 N^{\tau}}\left(\hat{g}_{\tau \tau}^{\prime \prime}-N^{\sigma} \hat{g}_{\tau \sigma}^{\prime \prime}+\left(N^{\sigma}\right)^{2} \hat{g}_{\sigma \sigma}^{\prime \prime}\right)-N^{\tau} T^{2} \hat{g}_{\sigma \sigma}^{\prime \prime}-T \hat{B}_{M N}^{\prime \prime} \partial_{\tau} \tilde{x}^{M} \partial_{\sigma} \tilde{x}^{N},
$$

where

$$
\hat{G}_{M N}^{\prime \prime}=\hat{G}_{M N}^{\prime}+\frac{2}{\mathbf{X}} \mathbf{M}_{M N}, \quad \hat{B}_{M N}^{\prime \prime}=\hat{B}_{M N}^{\prime}+\frac{1}{\mathbf{X}}\left(\mathbf{A}_{N} \mathbf{B}_{M}-\mathbf{A}_{M} \mathbf{B}_{N}\right) .
$$

Now we return to the background (2.21). In this explicit case the matrix $\hat{G}_{m n}$ has components

$$
\hat{G}_{m n}=\left(\begin{array}{cc}
h_{y y}-2 \tau_{y} m_{y} & \tau_{y} \\
\tau_{y} & \hat{G}_{u u}
\end{array}\right)
$$

so that inverse metric has the form

$$
\tilde{G}^{m n}=\frac{1}{\operatorname{det} \hat{G}_{m n}}\left(\begin{array}{lc}
\hat{G}_{u u} & -\tau_{y} \\
-\tau_{y} & h_{y y}-2 \tau_{y} m_{y}
\end{array}\right),
$$

where

$$
\operatorname{det} \hat{G}_{m n}=\left(h_{y y}-2 \tau_{y} m_{y}\right) \hat{G}_{u u}-\tau_{y} \tau_{y} .
$$

As it is clear from the matrix above the condition to have $\hat{G}^{\eta \eta}=\frac{1}{\hat{G}_{u u}}$ we should demand that $\tau_{y}=0$. so that

$$
\tilde{G}^{m n}=\left(\begin{array}{cc}
\frac{1}{h_{y y}} & 0 \\
0 & \frac{1}{\hat{G}_{u u}}
\end{array}\right) .
$$

In this case we obtain following components of the vectors $\mathbf{A}_{M}$ and $\mathbf{B}_{M}$

$$
\mathbf{A}_{M}=\left(-\frac{\hat{G}_{i u}}{\sqrt{\hat{G}_{u u}}}, 0, \frac{1}{\sqrt{\hat{G}_{y y}}}\right), \quad \mathbf{B}_{M}=\left(\frac{\hat{G}_{i u}}{\sqrt{\hat{G}_{u u}}}, 0, \frac{1}{\sqrt{\hat{G}_{y y}}}\right)
$$


and hence matrix $\mathbf{M}_{M N}$ has the form

$$
\begin{aligned}
& \mathbf{M}_{i j}=-\frac{\tau_{i} \tau_{j}}{\hat{G}_{u u}}, \quad \mathbf{M}_{i \tilde{y}}=0, \quad \mathbf{M}_{i \eta}=\tau_{i}, \\
& \mathbf{M}_{\tilde{y} \tilde{y}}=\mathbf{M}_{\tilde{y} \eta}=0, \quad \mathbf{M}_{\eta \eta}=\frac{1}{\hat{G}_{u u}} .
\end{aligned}
$$

When we choose $\hat{G}_{u u}=1$ we obtain that T-dual background fields have the form

$$
\begin{aligned}
\hat{G}_{i j}^{\prime} & =\hat{h}_{i j}-\frac{\hat{h}_{i y} \hat{h}_{y j}}{h_{y y}}-\tau_{i} \tau_{j}-\frac{\hat{B}_{i y} \hat{B}_{y j}}{h_{y y}} \equiv \hat{h}_{i j}^{\prime}-\tau_{i} \tau_{j} \\
\hat{G}_{i} \tilde{y} & =-\frac{1}{h_{y y}} \hat{B}_{y i}, \quad \hat{G}_{i}^{\prime} \tilde{y}=\frac{1}{h_{y y}} \hat{B}_{i y}, \\
\hat{G}_{i}^{\prime \eta} & =-\hat{B}_{u i}=0, \quad \hat{G}_{i}^{\prime \eta}=\hat{B}_{i u}=0 \\
\hat{G}^{\prime \eta \eta} & =1, \quad \hat{G}^{\prime \tilde{y} \tilde{y}}=\frac{1}{h_{y y}} \\
\hat{B}_{i j}^{\prime} & =\hat{B}_{i j}-\frac{\hat{B}_{i y} \hat{G}_{y j}}{h_{y y}}-\hat{B}_{i u} \tau_{j}+\tau_{i} \hat{B}_{u j}+\frac{\hat{G}_{i y} \hat{B}_{y j}}{h_{y y}}=\hat{B}_{i j}-\frac{\hat{B}_{i y} \hat{h}_{y j}}{h_{y y}}+\frac{\hat{h}_{i y} \hat{B}_{y j}}{h_{y y}} \\
\hat{B}_{i}^{\prime} \tilde{y} & =\frac{\hat{G}_{i y}}{h_{y y}}=\frac{\hat{h}_{i y}}{h_{y y}}, \quad \hat{B}_{i}^{\prime \eta}=\tau_{i} .
\end{aligned}
$$

It is instructive to find explicit form of the T-dual Lagrangian. First of all we have

$$
\mathbf{M}_{\alpha \beta}=\left(\partial_{\alpha} \eta-\tau_{\alpha}\right)\left(\partial_{\beta} \eta-\tau_{\beta}\right) .
$$

Then we obtain

$$
\begin{aligned}
g_{\alpha \beta}^{\prime} & =\hat{h}_{\alpha \beta}^{\prime}-\tau_{\alpha} \tau_{\beta}+\partial_{\alpha} \eta \partial_{\beta} \eta, \\
\hat{B}_{M N}^{\prime} \partial_{\tau} \tilde{x}^{M} \partial_{\sigma} \tilde{x}^{N} & =\hat{B}_{\tau \sigma}^{\prime}+\tau_{\tau} \partial_{\sigma} \eta-\tau_{\sigma} \partial_{\tau} \eta
\end{aligned}
$$

and hence we see that T-dual Lagrangian density has the same form as in (2.27) (with zero $b_{\mu}$ ) with background metric and NSNS two form fields given in (3.26). This is again nice consistency check. Of course we should stress that we do not consider the most general case when $\hat{B}_{u \mu}=b_{\mu} \neq 0$. On the other hand we do not expect that the presence of non-zero NSNS two form field qualitatively changes the transformation rules presented in this paper however this problem should be investigated further.

As the last remark we would like to discuss relation between our results and T-duality transformations of stringy non-relativistic string which was analysed in [9]. As was shown there T-duality of stringy non-relativistic string along longitudinal spatial direction leads to relativistic string in the background with light-like isometry which is exactly the action we started with. Then we argued that T-duality of non-relativistic string in torsional NC background corresponds to T-duality along two directions in relativistic string with lightlike isometry, one which is light-like and the second one which is spatial one. Clearly this T-duality is the same as T-duality along transverse direction in stringy non-relativistic string theory [9]. We mean that this again nice consistency check of our approach. 
Open Access. This article is distributed under the terms of the Creative Commons Attribution License (CC-BY 4.0), which permits any use, distribution and reproduction in any medium, provided the original author(s) and source are credited.

\section{References}

[1] R. Andringa, E. Bergshoeff, S. Panda and M. de Roo, Newtonian Gravity and the Bargmann Algebra, Class. Quant. Grav. 28 (2011) 105011 [arXiv:1011.1145] [INSPIRE].

[2] T. Harmark, J. Hartong and N.A. Obers, Nonrelativistic strings and limits of the AdS/CFT correspondence, Phys. Rev. D 96 (2017) 086019 [arXiv:1705.03535] [InSPIRE].

[3] T. Harmark, J. Hartong, L. Menculini, N.A. Obers and Z. Yan, Strings with Non-Relativistic Conformal Symmetry and Limits of the AdS/CFT Correspondence, JHEP 11 (2018) 190 [arXiv: 1810.05560] [INSPIRE].

[4] R. Andringa, E. Bergshoeff, J. Gomis and M. de Roo, 'Stringy' Newton-Cartan Gravity, Class. Quant. Grav. 29 (2012) 235020 [arXiv:1206.5176] [INSPIRE].

[5] E.A. Bergshoeff, J. Gomis, J. Rosseel, C. Şimşek and Z. Yan, String Theory and String Newton-Cartan Geometry, J. Phys. A 53 (2020) 014001 [arXiv:1907.10668] [InSPIRE].

[6] J. Klusoň and P. Novosad, Non-Relativistic M2-Brane, JHEP 06 (2019) 072 [arXiv: 1903.12450] [INSPIRE].

[7] J. Klusoň, $(m, n)$-String and D1-Brane in Stringy Newton-Cartan Background, JHEP 04 (2019) 163 [arXiv: 1901.11292] [INSPIRE].

[8] E.A. Bergshoeff, K.T. Grosvenor, C. Simsek and Z. Yan, An Action for Extended String Newton-Cartan Gravity, JHEP 01 (2019) 178 [arXiv:1810.09387] [INSPIRE].

[9] E. Bergshoeff, J. Gomis and Z. Yan, Nonrelativistic String Theory and T-duality, JHEP 11 (2018) 133 [arXiv: 1806.06071] [InSPIRE].

[10] J. Klusoň, Nonrelativistic String Theory $\sigma$-model and Its Canonical Formulation, Eur. Phys. J. C 79 (2019) 108 [arXiv:1809.10411] [InSPIRE].

[11] J. Klusoň, Remark About Non-Relativistic String in Newton-Cartan Background and Null Reduction, JHEP 05 (2018) 041 [arXiv: 1803.07336] [INSPIRE].

[12] J. Klusoň, Hamiltonian for a string in a Newton-Cartan background, Phys. Rev. D 98 (2018) 086010 [arXiv: 1801.10376] [INSPIRE].

[13] J. Klusoň, Note about Hamiltonian formalism for Newton-Cartan string and p-brane, Eur. Phys. J. C 78 (2018) 511 [arXiv:1712.07430] [InSPIRE].

[14] J. Gomis, J. Oh and Z. Yan, Nonrelativistic String Theory in Background Fields, JHEP 10 (2019) 101 [arXiv: 1905.07315] [INSPIRE].

[15] A.D. Gallegos, U. Gürsoy and N. Zinnato, Torsional Newton Cartan gravity from non-relativistic strings, arXiv:1906.01607 [INSPIRE].

[16] T. Harmark, J. Hartong, L. Menculini, N.A. Obers and G. Oling, Relating non-relativistic string theories, JHEP 11 (2019) 071 [arXiv:1907.01663] [INSPIRE].

[17] J. Klusoň, Note About Canonical Description of T-duality Along Light-Like Isometry, Eur. Phys. J. C 80 (2020) 103 [arXiv:1905.12910] [INSPIRE]. 
[18] T.H. Buscher, A Symmetry of the String Background Field Equations, Phys. Lett. B 194 (1987) 59 [INSPIRE].

[19] T.H. Buscher, Path Integral Derivation of Quantum Duality in Nonlinear $\sigma$-models, Phys. Lett. B 201 (1988) 466 [INSPIRE].

[20] E. Alvarez, L. Álvarez-Gaumé and Y. Lozano, A Canonical approach to duality transformations, Phys. Lett. B 336 (1994) 183 [hep-th/9406206] [INSPIRE].

[21] E. Alvarez, L. Álvarez-Gaumé and Y. Lozano, An Introduction to T duality in string theory, Nucl. Phys. Proc. Suppl. 41 (1995) 1 [hep-th/9410237] [InSPIRE].

[22] J. Klusoň, Note About T-duality of Non-Relativistic String, JHEP 08 (2019) 074 [arXiv: 1811.12658] [INSPIRE].

[23] J. Klusoň, Non-Relativistic D-brane from T-duality Along Null Direction, JHEP 10 (2019) 153 [arXiv: 1907.05662] [INSPIRE].

[24] J. Gomis and H. Ooguri, Nonrelativistic closed string theory, J. Math. Phys. 42 (2001) 3127 [hep-th/0009181] [INSPIRE].

[25] U.H. Danielsson, A. Guijosa and M. Kruczenski, IIA/B, wound and wrapped, JHEP 10 (2000) 020 [hep-th/0009182] [INSPIRE]. 